\title{
Diabetes-related communications in community pharmacy: reflections on the findings from a feasibility study
}

Nathalie Haigh Research Consultant for Wolds Primary Care Research Network (WoReN) and Hull \& East Riding Pharmacy Research Network (HERPRN), UK, Peter Campion Foundation Professor in Primary Care, The Post Graduate Medical Institute at the University of Hull, UK, Valerie Featherstone Research Facilitator of WoReN, UK, Hilary Edmondson Lead of HERPRN, UK, Koon Hien Tee Head of Operations, Independent Pharmacy Care Centres plc, UK and Andrew Innes General Practitioner/researcher in primary care and Chair of WoReN, UK

\begin{abstract}
Aims: To assess the feasibility of keeping a log of diabetes-related communication in a community pharmacy and to explore the potential of the logs to reflect diabetes-related communications within pharmacies. Design: A reflective log-keeping exercise (the log designed by the researchers and provided in a booklet form) in community pharmacies of any diabetes-related communication taking place between pharmacy staff and their service users, and any signposting made to and from other relevant health professionals. The study ran for four weeks. Subjects and setting: Nine community pharmacy branches of the Independent Pharmacy Care Centres PLC based in Hull, East Yorkshire and North \& North East Lincolnshire. Outcome measures: A description of the form and nature of diabetes-related communication, taking place within the community pharmacy. The research team also sought feedback on the experience of keeping the log. Results: Twenty-two communications were logged; twenty-one involved direct face-toface contact and one log involved a telephone conversation. Two-thirds of the communications involved conversations with patients with diabetes, a third were with a partner/family member. The conversations captured fell into five categories: sugar free medicines, blood glucose-testing metre, prescription medicines, diabetes specific education/information and an unclassified category, respectively. The logs demonstrated that communications can involve all members of the staff team, working independently or jointly across their roles in the pharmacy. Conclusion: Community pharmacies in the pilot study were actively involved in providing diabetes health-related information/education and support. Communications involved directly assisting people with diabetes and providing information to people supporting others with a diagnosis of diabetes. We propose that the logs provide a valuable means of acquiring information about the form and nature of diabetes-related communication and signposting within community pharmacy.
\end{abstract}

Key words: brief interventions; carer support; community pharmacy; diabetes communications; Diabetes National Service Framework; log keeping; self-care; service development; The New NHS Community Pharmacy Contract

Received: June 2006; accepted: November 2006

Address for correspondence: Prof Peter Campion, Post Graduate Medical Institute, Psychological and Primary Care Medicine, Room 306, Hertford Building, University of Hull, Cottingham Road, Hull HU6 7RX, UK. Email: p.d.campion@hull.ac.uk

(C) 2007 Cambridge University Press 


\section{Introduction}

This article reflects on the findings from a small study, examining the feasibility of keeping a log of diabetes-related communication in community pharmacy. The study was undertaken as a precursor for part of a larger research project, which aims to explore the perceptions and experiences of community pharmacy diabetes care provision: those of service users, their local pharmacists and other health professionals involved in diabetes care. This article considers the results of the feasibility study in relation to The National Service Framework for diabetes (DNSF, Department of Health, 2001) and the developing role of community pharmacy in disease management.

Diabetes is one of the most significant public health challenges facing the UK today (Diabetes UK, 2005). The government is now determined to decrease the burden of diabetes across the National Health Service (NHS) in primary care, acute hospitals and community services (Department of Health, 2001). As a life-long disorder, diabetes has the potential significance to influence all aspects of life from health, well-being, life expectancy, through to lifestyle, relationships, work and income. The effective management of diabetes can reduce complications and increase life expectancy. Living with diabetes is not straightforward: it involves personal commitment and an active involvement with the condition. People with diabetes manage their condition on a day-to-day basis within their lifestyles and circumstances, away from the care and instruction of health care professionals. Ninety-five per cent of the management of diabetes is self-care (Diabetes UK, 2005). Thus, the basis of good diabetes care is to empower patients to play a central role in the management of their disorder (The St Vincent Declaration, 1990).

The National Service Framework programme (NSF) was established to set national standards in the NHS in an attempt to improve service provision and equity in care (Department of Health, 2002a). The DNSF puts forward a long-term plan to provide better care for people with diabetes. The aim is to care for people with diabetes using patientcentred service models (Department of Health, 2001, 2004a). The DNSF suggests that primary health care teams should determine the best way of delivering the diabetes standard by considering the proposals outlined in the Delivery Strategy in terms of their own local circumstances and the communities which they serve. At the same time, the government is calling on pharmacists to develop more responsive and innovative services to meet diabetes NSF targets. With their local knowledge of the local community, community pharmacists are well placed to regularly interact meaningfully with patients to deliver health promotion and medicines use (Department of Health, 2003).

The DNSF requires pharmacists (both individually and collectively) to reconsider their role in diabetes care in the multidisciplinary diabetes care team. The potential of community pharmacists of being instrumental in the implementation of the DNSF standards and objectives is demonstrated clearly in terms of Type 2 diabetes: community pharmacists can dispense medication, provide inhouse medication management (including a medicines use review) (Department of Health, 2003; The Pharmaceutical Services Negotiating Committee, 2004a) and a diabetes monitoring service (including blood glucose blood pressure, blood lipid, and HbA1c); screen for undiagnosed diabetes; and provide information to help people with diabetes to understand their disorder. In their role in health promotion, pharmacists can actively promote exercise and provide advice on diet, smoking cessation and healthy lifestyles by providing over-the-counter counselling and various in-house programmes (Royal Pharmaceutical Society of Great Britain, 2006). This expansion of their role could feasibly include the proposed enhanced care for people with diabetes.

The new NHS Community Pharmacy Contract (The Pharmaceutical Services Negotiating Committee, 2004b) and numerous other major developments in the NHS in 2003-2004, which have been designed to compliment each other, have wide implications for future developments in community pharmacy. These include, among others, the continued devolution of central funding to Primary Care Trusts, the agreement on the new General Medical Services (GMS) contract for general practitioners (GPs) (NHS Confederation and British Medical Association (BMA), 2003; BMA, 2006/ 2007) Medicines Management (Department of Health, 2004b) and Chronic Disease Management schemes (Department of Health, 2002b). Pharmacists have both the accessibility and expertise to significantly contribute to improving medicines management, health promotion and the self-management 
of chronic disease (Department of Health, 2000, 2002b, 2003; Royal Pharmaceutical Society of Great Britain, 2006). The new contract for community pharmacy (Pharmaceutical Services Negotiating Committee, 2004b) specifically requires pharmacists to record advice given on prescribed medication and self-care; to undertake appropriate health promotion and to 'signpost' to other sources of help.

The Quality and Outcomes Framework (2004), part of the new GMS contract for GPs, has standards and specific targets set for diabetes, along with other chronic disorders. General practices are being paid for meeting targets, including their participation in data collection of disease-related health indicators, on the basis of a points system, determined through the provision of good quality services. This flexibility in meeting set targets creates opportunities for pharmacists, who may be commissioned by general practice to help achieve the standards set in the contract ${ }^{1}$. Medicines Management is a major government initiative: a strategy for the clinical, cost-effective and safe use of medicines to ensure that patients receive the maximum benefit from the medicines they require, whilst also minimizing potential harm. The primary prevention of chronic disease management strategy also includes diabetes as a risk factor. Self-management at an early stage may help prevent the onset of complications and further disability (Department of Health, 2002b).

The Department of Health is considering how the role of pharmacy may be enhanced to improve support for people with diabetes. The implementation of the DNSF programme in community pharmacies will affect working practice and will require effective organization based on best current practice. The new NSF health care standards will revise contractual arrangements and service-planning issues affecting community pharmacy practice. The changes may not be easy and therefore it is important to assess whether the proposed role of the community pharmacies in the new DNSF is workable, acceptable (to pharmacists and their customers) and cost-effective at a local (and national) level. Many of the early studies investigating the role of community pharmacists in the management

\footnotetext{
${ }^{1}$ For instance, pharmacists could develop enhanced services such as smoking cessation clinics and carry out medication reviews to challenge concordance and waste (Royal Pharmaceutical Society of Great Britain, 2004).
}

of diabetes were either conducted in America or hospital-based, and thus not entirely transferable to community-based services in the UK (Cantril, 2004). Studies that have considered the pharmacist's role in the management of diabetes in the UK demonstrate that pharmacists, working as part of a multidisciplinary team, can improve diabetes management in patients (Coast-Senior et al., 1998; Pickard et al., 1999; McClean et al., 2000; Bliss et al., 2001; Rajaei-Dehkordi et al., 2003; Wermeille et al., 2004). However, further research is required and future interventions need to integrate evidence from the literature on patient and pharmacist perspectives on diabetes (Blenkinsopp and Hassey, 2005).

The diabetes communication log is the first part of a three-stage study designed by the Hull \& East Riding Pharmacy Research Network (HERPRN) and the Wolds Primary Care Research Network (WoReN) exploring the perceptions and experiences of community pharmacy in diabetes care provision: those of service users, their local pharmacists and other health professionals involved in diabetes care ${ }^{2}$. The purpose of the log-keeping exercise in this study is to capture the form and nature of diabetes-related communication taking place within the community pharmacy.

\section{Method}

\section{Log design}

The log was designed by the researchers with the help of local community pharmacists to record any communication related to diabetes, including medication, advice, signposting and referrals ('advice' was defined as any verbal interaction which involved more than simply a financial transaction, Bissell et al., 1997). The log consisted of 30 identical double-sided pages of A4 with the following sections:

1) The role of the pharmacy staff member filling in the form (tick box).

2) How the communication reported took place (tick box), and with whom (tick box and add details: 'customer with diabetes', 'health professional', 'other representative', and an 'other' option).

\footnotetext{
${ }^{2}$ See http://www.woren.org/projects.html

Primary Health Care Research \& Development 2007; 8: 147-156
} 
3) Whether the customer/professional had been 'signposted/recommended' to the pharmacy and by whom (tick box and add details).

4) A free-text description of the communication, how it came about and what was said by all parties.

5) Any action taken or other result of the communication (free text).

6) Whether any written information, such as leaflets were provided (Yes/no with details).

7) Whether the communication resulted in 'signposting' the person concerned to another person. (Yes/no with details)

\section{Pre-pilot work}

In preparation for the study, the authors involved the four funded research pharmacies of the East Riding Pharmacy Research Network for comments and suggestions about the form and layout of the log and its contents, and feedback on the experience of keeping the $\log$.

\section{Setting}

We chose to use for this pilot study a group of independent community pharmacies based in Hull, East Yorkshire and North Lincolnshire ${ }^{3}$. This was in effect a convenience sample, but it represented a range of local pharmacies, both urban and rural, not part of a large chain, which had not previously been involved in research, and so were more representative of the wider community than those engaged in the pharmacy research network.

\section{Data collection}

A letter, authorized by the Head of Operations of the IPCC chain, was sent out to the pharmacies involved. This explained what the feasibility study was about, how it related to the proposed study and their proposed role in piloting the communication log. The letter was followed a week later by a telephone call, contacting the 'appointed person'

\footnotetext{
${ }^{3}$ Independent Pharmacy Care Centres plc (IPCC).

Primary Health Care Research \& Development 2007; 8: 147-156
}

in the pharmacies involved, to confirm the best time and date of the delivery. A second letter was sent, confirming the arrangements. Members of the research team personally delivered the logs to the pharmacies, explained the logs and how to complete them. The logbook contained instructions, two examples and evaluation sheets to be completed at the end of the study. The log instructions explained that the exercise was not intended to evaluate the service provided by the pharmacy. Rather the log had been specifically designed to help researchers to gain an insight into the everyday communication taking place in pharmacies relating to any aspect of diabetes care.

The feasibility study was initially planned to run for three weeks in total, but when some pharmacists indicated that they had not completed a sufficient number of logs, we decided to continue for a further week. Completed logs were returned to the researchers with a prepaid stamped and addressed envelope.

\section{Data analysis}

The diary entries were coded in relation to the seven main questions comprising the log (listed 1-7 above). The researcher (NH) made a tally of the number of responses that fell under each of the set categories (questions 1, 2, 3, 6 and 7) and created descriptive categories for the open responses (questions 3, 4, 5, 6 and 7). Some closed questions offered the respondent an opportunity to provide further description. For example, in question 3, where the customer/professional was signposted to the pharmacy (involving a yes or no response), the respondent is invited to state by whom. Responses to questions 4 and 5, reason for the communication and the outcomes of the communication, were initially categorized from the data then collapsed into higher order categories as they emerged. In this article, the outcomes of communication are presented descriptively after presenting the reasons for the communication to give the reader more content of brief interventions in the ensuing communication. For instance, not all outcomes simply involved the sale of a new blood glucose-testing meter (BGTM).

The contents of the logs were categorized by NH and verified and agreed by a second researcher, VF. The findings from the data are outlined below. 
Table 1 Variation of staff involved in communications

- Regular pharmacist $(12)^{\mathrm{a}}$

- Pharmacy technician (4)

- Counter assistant/pharmacy technician (3)

- Regular pharmacist, joint with dispenser (2)

- Locum pharmacist (1)

a Numbers in parenthesis indicating the number of logs completed by designation.

\section{Findings}

Twenty-two log sheets were completed over the four-week period in nine pharmacies. The average number of completed logs per pharmacy was two (a range of 1-5 log sheets). The median length of time to complete a log was $5 \mathrm{~min}$, the range being $3 \mathrm{~min}$ to $4 \mathrm{~h}^{4}$. Whilst the regular pharmacist made most entries, the logs demonstrated that diabetesrelated communications can involve all members of the staff team, working independently or a jointly across their roles in the pharmacy. Table 1 outlines the variation of staff involved in communications related to diabetes.

Two-thirds of the communications involved conversations with patients with a diagnosis of diabetes while one-third involved a patient's representative. We shall return to the significance of this later. The type of diabetes was insulin-dependent in five instances, Type 2 (non-insulin dependent) in 13, and in four the type was not recorded.

Twenty-one of the communications involved direct face-to-face contact with the customer; one involved a telephone conversation. Pharmacy staff initiated three of the face-to-face diabetes-related conversations by picking up on cues in conversation and by asking questions. Two of these communications involved the purchase of sugar free cough medicines and the third involved the sale of a new BGTM.

None of the communications logged involved direct communication with another health professional. One customer was signposted to the pharmacy by their GP to purchase a glucose-testing meter.

The conversations captured fell into five categories: sugar free medicines, BGTM, prescription medicines, diabetes specific education/information

\footnotetext{
${ }^{4}$ One log took $4 \mathrm{~h}$ to complete due to constant interruption.
}

Table 2 The categories of communication

1) Sugar-free medicines $(7)^{\mathrm{a}}$

2) BGTM (7)

3) Prescription medicines (4)

4) Diabetes specific education/information (3)

5) Unspecific (1)

a Numbers in parenthesis indicating the number of logs completed per category of communication.

and an unclassified category, respectively. Table 2 shows the categories of communications.

1) Sugar free medicines. These communications included advice on: (a) Pholcodine Linctus for partner with diabetes (a sugar free version was purchased and this was initiated by the pharmacist); (b) a query about Lemsip (sugar free alternative offered); (c) the best cough medicine for people with diabetes; (d) cough medicine for diabetes; (e) cough medicine (sugar free version, conversation initiated by pharmacist); (f) a query about sugar in Strepsils and (g) sugar free throat lozenges (customer also worried and confused over new diagnosis and perceived lack of information from main health care provider).

A sugar free product was sold in all cases. Enquiries about sugar free medicines provide pharmacists opportunities to impart general diabetes health promotion. For example, one communication was about controlling the intake of sugar in their diet, another enquired about current glucose readings, where the pharmacist suggested more regular checks during periods of illness.

2) BGTM. These were a referral by a GP to buy a glucose-testing meter; a man seeking to purchase a BGTM for his wife who was newly diagnosed; seeking advice about buying a BGTM; looking to buy batteries for new BGTM; seeking advice about ordering new test strips for BGTM; purchasing a new BGTM (conversation initiated by pharmacist) and ordering a new insulin pen (the customer was confused about his prescription).

Blood glucose-testing kits were sold in three of these encounters, and a meter was exchanged in another. Two gave information about the pharmacy's HbA1c monitoring scheme. One of these logs also noted informing the patient about free NHS prescriptions. Another included information about how best to use the BGTM; the 
patient's knowledge about acceptable blood glucose levels was also checked. The remaining three communications resulted in the pharmacist providing contact details of a supplier (a set of batteries loaned to the customer meanwhile); giving information on ordering new test strips for BGTM and solving the confusion over a prescription and the dispensing of the correct insulin pen.

3) Prescription medicines. This category included (a) a woman noting her partner's recent change to insulin and concerns about his alcohol intake (the conversation took place with the pharmacists whilst she picking up methadone for her son); (b) a newly diagnosed patient confused about the dosage stated on his prescription, expressing worries about his eyesight; (c) a customer seeking more information about contents of a new prescription and (d) a query as to whether partner can drink alcohol with his new medication.

Information about insulin and diabetes was given to the woman concerned about her husband who has diabetes. She stated that she intended to encourage her husband to take on a healthier lifestyle. In the case of the confused newly diagnosed patient, information was given about new tablets and the dosage in the prescription. Reassurance was offered along with diabetes-related information and the importance of adherence to medication was stressed. Relevant information was provided to the individual who queried the prescription and diabetes-related information/education was also offered. The pharmacist advised the woman that her husband could drink alcohol in moderation when taking his medication. However, this advice was expanded upon by further explanation of the effect of alcohol on blood pressure and the implications of high blood pressure for levels of blood glucose.

4) Diabetes specific education/information. A woman was reassured about using flight socks and given related information focusing on the health risks related to diabetes and general health advice. Another customer in conversation with the regular pharmacist whilst dispensing a prescription did not require any specific information, but there was a general discussion about diabetes. A third customer was worried about her (diabetic) partner's health/behaviour and change in mood. She was given health information on

\section{Box 1 Example of free-text entries in one log}

Tell us about the communication, how it came about, what was said by all parties, etc.

'The customer came in with a new item on the prescription. He was confused with the dosage instruction given by the doctor for this hypoglycaemic tablet. I explained to him what the dosage instruction means and showed him what the BNF said as well. He is a newly diagnosed diabetic, so I asked him if he has got another appointment either with the surgery or diabetic clinic to get the blood glucose measured after he started the treatment. He is not quite sure when but will need to go back to see the GP after a month. Fasting glucose level is $\sim 8 \mathrm{mmol} / \mathrm{L}$.'

What action was taken/what was the result of the communication?

'Customer was worried about his eye sight. Explained to him to get his eyes checked regularly should keep him up-to-date with his condition. But assured him that when treatment started at the early stage of the condition, it should slow down the progression of the disease. Convinced him that he should take the tablets as prescribed and monitor his blood glucose.'

diabetes, the symptoms of diabetes were also explained and she was advised to encourage her husband to visit the diabetes nurse.

5) Unclassified category. This involved a lady with diabetes who sought advice about the best painkillers to take for her sore knee. Painkillers were sold and relevant health information was provided.

Three logs described the provision of written information to patients. Information leaflets/manuals were provided with the BGTM on two occasions, and another described an instance where the pharmacists talked a customer through written information on diabetes and provided an article to take away (her husband was newly diagnosed).

\section{Discussion}

This small feasibility study captured some day-today examples of the essential services outlined in 
the new NHS pharmacy contract (NHS Confederation, 2004). The study demonstrated that pharmacists were providing over-the-counter counselling on medication, diabetes disease management, the reduction of complications, promoting healthy lifestyles and signposting customers (patients and their carers), where necessary. The two essential services, promotion of healthy lifestyles and support for self-care (Royal Pharmaceutical Society of Great Britain, 2006), were particularly noted.

The provision of information, education and psychological support that promotes self-management is the cornerstone of diabetes care (Department of Health, 2001). These NSFD Standards (Department of Health, 2001) states that a significant proportion of people with diabetes do not understand the basic principles of their diabetes care. People with diabetes require the knowledge, skills and motivation to evaluate their health risks, to appreciate how they will benefit from changing their behaviours or lifestyle and to act on this knowledge by engaging in appropriate behaviours (Department of Health, 2001). Several communications logged in the current study involved attending to confusion and concern in people newly diagnosed with diabetes, for whom pharmacy staff offered reassurance and advice on the importance of adherence to their medication plan. It has been suggested that pharmacists are well placed to promote positive health behaviour because they are often likely to be the last health care professional to have contact with patients prior to them taking their medication (Morrow and Hargie, 1994; Rees, 1996). The pharmacist can remind, reinforce and extend the education of its customers with diabetes, evaluate individual knowledge of the condition and ensure treatment concordance (Royal Pharmaceutical Society of Great Britain, 2004). This role was evident in the communications logged in the current study. As Diabetes NSF standards become incorporated in pharmacy care service provision, pharmacists need to continue to demonstrate this role for themselves and develop this within local primary care communities.

The new contractual framework for community pharmacy (The Pharmaceutical Services Negotiating Committee, 2004a) and the recent White Paper on Community Services (Department of Health, 2006) provide opportunity for community pharmacists to play a central role in delivering improvements in primary care. The long-term conditions agenda is one of the government's principle initiatives.
Pharmacists aim to support self-care (of long-term conditions) in three ways: proactive self-care, facilitated self-care and supported self-care (Royal Pharmaceutical Society of Great Britain, 2006). For pharmacists, self-care should not be just about medicines - it is also about engaging with the public and transforming to a patient-centred pharmacist role (Royal Pharmaceutical Society of Great Britain, 2006).

In the present pilot study, participating pharmacists actively imparted information, education and self-care advice about diabetes to customers in half of the communications captured. Customers sought advice from pharmacy staff, asking both direct and indirect questions about diabetes management. It is the role of pharmacy staff to reflect on and develop these interactions further, record them where they see fit and in the context of their work. Feedback from one pharmacist, who provided four log entries, highlighted that customers did not directly look to pharmacists for advice about their condition. She explained that the proactive approaches by staff could be uncomfortable for some customers because not all individuals welcomed unsolicited questions and comments. This pharmacist commented that discussions of disease states and management should be a matter of course in pharmacy service provision. Training can help change perceptions about offering proactive advice for those pharmacists (and their staff) who feel uncomfortable in this role (Department of Health, 2005). Where opportunity and time are available, efforts by pharmacy staff may serve to educate the public to accept, use and experience the pharmacy as a resource for health promotion/ education and general advice. For example, one log described a casual conversation between a customer and regular pharmacist where the customer did not require any further information. The same customer was invited to return for information should the occasion arise in the future. Informing and inviting customers to make use of services may be a small but significant example of the DNSF aspirations for wider and seamless support (in terms of accessible information and education) that can be offered to the local community.

The new pharmacy contract includes support for carers, the promotion of healthy lifestyles and support for self-care. It was clear from the logs, that communications between pharmacy staff and their customers not only involved assisting people

Primary Health Care Research \& Development 2007; 8: 147-156 
with diabetes, but also included the provision of information to family members of people with diabetes. The potential impact of supporting and educating carers about diabetes management in the current study is encouraging. For example, after inquiring after her husband's change in prescription one conversation progressed to diabetes management, and the customer said that she was going to encourage her husband to take on a healthier lifestyle. Showing interest and offering support and education to patients with diabetes (and in this case a family member) can boost motivation to help people deal with the demands of ongoing chronic management, and may also lead to behavioural change and improvement (Royal Pharmaceutical Society of Great Britain, 2004).

Signposting is an essential part of the new pharmacy contract (Royal Pharmaceutical Society of Great Britain, 2004; Department of Health, 2005). This is an important component given that it has been suggested that inaccessible and/or inadequate care provision may impose a barrier to diabetes care (Snoek, 2000). Community pharmacy has access (and is accessible) to other health professionals and thus can play a significant role in preventing and/or improving disease outcomes by advising their customers to consult other professionals in the diabetes health care team. They can also direct patients to local and national self-help groups such as patient advice and liaison services, and Diabetes UK, and put people in touch with personal health trainers (Department of Health, 2005). In our study, one customer was signposted to a diabetes nurse and two logs noted that membership of Diabetes UK had been advocated. It was apparent from the logs that where more professional input was required (such as an appointment at the diabetes clinic or the diabetes nurse), pharmacists checked with the patient whether appropriate arrangements were in place, or were in the process of being made. In this way the patient was reminded of the significance of attending health appointments and the community pharmacy is then experienced as part of the extended diabetes care team. Currently there is no formal system of referral from community pharmacy and this may be an area worthy of further exploration.

The availability of time to engage in log keeping was cited as a limitation to its wider use. However the new Pharmacy Contract requires pharmacists to record signposting, health promotion advice and interventions related to medication (Royal
Pharmaceutical Society of Great Britain, 2004). The log-keeping methodology allowed researchers to gain an insight into pharmacy-based communications that they cannot readily observe. Such communications may not be amenable to observation because they have no time boundaries, can be unpredictable, are private (and hence require confidentiality and respect) and are likely to be altered by the presence of an observer. Whilst the data derived from this small feasibility study was subject to a simple content analysis, it is possible to use these analyses to reflect on the value of log keeping in researching (specific disease-related) communication in community pharmacy. The completed logs helped illuminate the format and contexts within which the diabetes-related communications took place. Perhaps such an exercise can be used to remind pharmacy staff and their commissioners of the importance of what is 'taken for granted' in day-to-day communications within community pharmacy. The open format for questions 4 and 5 proved particularly useful for capturing communications within a context, particularly in recording how the pharmacy staff-customer interactions can develop opportunities for further/new discussion, leading on to further brief health interventions. The benefit of log keeping is the potential to record events, as close as possible to when they occur (see Box 1). Retrospective reframing is minimized. Details may be lost in a retrospective interview and specific detail is even more difficult to capture in a standard questionnaire, designed to reflect upon one's overall practice. The open format for questions 4 and 5 allowed informants some latitude to write about and to structure entries, as they felt appropriate. With the above points in mind, logkeeping periods may serve as both a record of interactions and a reflective exercise for the pharmacy team. One pharmacist involved stated that the exercise was salutary in reminding her and the pharmacy team to focus on different aspects of their work. It is important to note that the logs were commissioned specifically for research and therefore were not 'private' documents. They were written with the research team in mind, not as, for instance, a self-appraisal document for reflective practice. Perhaps as a reflective exercise, as well as revealing what interventions pharmacists made, recording disease specific communications would allow respondents the opportunity to reflect upon what they did not consider or do. For instance, it 
has been suggested that pharmacists and their staff should play closer attention to the self-care interventions that they undertake; they should ask themselves at every consultation: what step they can take to support the patient to better selfcare (Royal Pharmaceutical Society of Great Britain, 2006). Thus log keeping may form part of a research process, whereby pharmacy staff actively participate in both recording and reflecting upon their own practice, individually and/or as a team.

The recorded communications obtained in this small pilot study provides some insight into the day-to-day diabetes care services and support provided by community pharmacy. Such a log-keeping exercise may serve to bring to the foreground, diabetes-related communications that may be taken as a matter of course. The study confirmed that the logs are feasible and the information they acquire will be a valuable component in the proposed study. Whilst this feasibility study offers some hope that community pharmacists are actively involved in providing diabetes-related information/education and self-care support to their customers, the limitations of small numbers herein are acknowledged. Researchers were reminded that a lot more paperwork has been introduced under the New Pharmacy Contract. Keeping a log becomes an additional task within an increasing workload, and this pressure needs to be accounted for in a larger study or where a reflective log-keeping exercise is undertaken as part of service development.

\section{Conclusion}

Whilst this small pilot study involved only nine participating pharmacies, the logs do appear to be a feasible way of acquiring information about the form and nature of diabetes-related communication taking place within community pharmacy. The completed logs demonstrate that the pharmacists and staff participating in the study were actively involved in providing diabetes-related information/education and support to their customers. Customers sought advice from pharmacy staff, asking both direct and indirect questions about diabetes management. Communications involved directly assisting people with diabetes and the provision of information to people supporting those with a diagnosis of diabetes. The conversations captured fell into five categories: sugar free medicines, BGTM, prescription, diabetes specific education/information and an unclassified category, respectively. The logs demonstrate that communications can involve all members of the staff team, working independently or jointly across their roles in the pharmacy.

\section{Acknowledgements}

HERPRN and WoReN partly funded this feasibility study. The authors would also like to thank the Insulin Dependent Diabetes Trust for their contribution. The authors are grateful to pharmacists and staff of IPCC: Booths Pharmacy and Whistler Pharmacy in Hull; Old Town Chemist and Flamborough Pharmacy in Bridlington; Market Place Pharmacy in Hornsea; Bradley Pharmacy and Laceby Pharmacy in Grimsby, Moores Pharmacy and Berkeley Pharmacy in Scunthorpe.

\section{References}

Blenkinsopp, A. and Hassey, A. 2005: Effectiveness and acceptability of community pharmacy-based interventions in type 2 diabetes: a critical review of intervention design, pharmacist and patient perspectives. The International Journal of Pharmacy Practice 13, 231-40.

Bissell, P., Ward, P.R. and Noyce, P.R. 1997: Insights into the implementation of in-store protocols in community pharmacies. The Pharmaceutical Journal 259 (Suppl), R36.

Bliss, E., Codack, H. and Boothe, J. 2001: Diabetes care - an evaluation of a community pharmacy based HbA1c testing service. The Pharmaceutical Journal 267, 264-66.

British Medical Association. 2006/2007: Revisions to the GMS contract, 2006/07. Delivering Investment in General Practice. Retrieved 18 May 2006, from http://www.bma.org.uk/ ap.nsf/Content/revisionnGMSFeb20062.

Cantril, A. 2004: Evaluating pharmaceutical care in patients with diabetes in Royal Pharmaceutical Society of Great Britain. Diabetes task force. Practice guidelines for community pharmacists on the care of patients with diabetes, third edition. London: Royal Pharmaceutical Society of Great Britain, 29-31.

Coast-Senior, E.A., Kroner, B.A., Kelly, C.L. and Trilli, L.E. 1998: Management of patients with type 2 diabetes by pharmacists in primary care clinics. Annals of Pharmacotherapy 32, 636-41.

Department of Health. 2000: Pharmacy in the future - implementing the NHS plan. A programme for pharmacy in the National Health Service. London: The Department of Health. 
Department of Health. 2001: National Service Framework for Diabetes: Standards. London: The Department of Health.

Department of Health. 2002a: National Service Frameworks. A practical aid to implementation in primary care. London: The Department of Health.

Department of Health. 2002b: Chronic disease management and self-care. National Service Frameworks. A practical aid to implementation in primary care. London: The Department of Health.

Department of Health. 2003: A Vision for Pharmacy in the new NHS. London: The Department of Health.

Department of Health. 2004a: The NHS Improvement Plan. Putting people at the Heart of Public Services. London: The Department of Health.

Department of Health. 2004b: Management of medicines - a resource to support implementation of the wider aspects of medicines management for the National Service Frameworks for Diabetes, Renal Services and Long-Term Conditions. London: The Department of Health.

Department of Health. 2005: Choosing health through pharmacy. A programme for pharmaceutical public health 2005-2015. London: The Department of Health.

Department of Health. 2006: Our health, our care, our say: a new direction for community services. TSO (The Stationary Office, Norwich): Department of Health, 87-89.

Diabetes UK. 2005: Diabetes: State of the Nations 2005. Progress made in delivering the national diabetes frameworks. In partnership with the All-Party Parliamentary Group for Diabetes and supported by the Hansard Society. London: Diabetes UK.

McClean, M.T., McElnay, J.C. and Andrews, J. 2000: The importance of patient education and patient involvement in the treatment of diabetes. Pharmaceutical Journal 265 (Suppl), R20.

Morrow, N. and Hargie, W. 1994: Communication skills and health promotion. Pharmaceutical Journal 253, 311-13.

NHS Confederation and British Medical Association (BMA). 2003: Investing in General Practice. The New General Medical Services Contract. Retrieved 17 March 2006, from http://www.bma.org.uk/ap.nsf/Content/investinggp.

NHS Confederation. 2004: Community pharmacy: essential and advanced services. Community and pharmacy briefing.
Retrieved 10 May 2006 from, http://www.psnc.org.uk/ index.php?type $=$ page $\&$ pid $=107 \& \mathrm{k}=2$

Pickard, A.S., Johnson, J.A. and Farris, K.B. 1999: The Impact of Pharmacist Interventions on Health-Related Quality of Life. Annals of Pharmacotherapy 33, 1167-72.

Quality and outcomes framework. Guidance - updated 2004. Health and Social Care Information Centre. Retrieved 18 May 2006, from http://www.bma.org.uk/ap.nsf/Content/ QualityOutcomes.

Rajaei-Dehkordi, Z., Hollingshead, C., Herkes, D., Holden, M. and Rao, S. 2003: Investigating the contribution of community pharmacists in identifying, understanding and meeting the needs of patients with diabetes, in collaboration with other health care professionals. The International Journal of Pharmacy Practice 11 (Suppl), R18.

Rees, P.A. 1996: Counselling and pharmacists. Pharmaceutical Journal, 257, 659.

Royal Pharmaceutical Society of Great Britain. 2004: Diabetes task force. Practice guidelines for community pharmacists on the care of patients with diabetes, second edition. London: Royal Pharmaceutical Society of Great Britain.

Royal Pharmaceutical Society of Great Britain. 2006: The self care challenge. A strategy for pharmacists in England. London: Royal Pharmaceutical Society of Great Britain.

Snoek, F.J. 2000: Barriers to good glycaemic control: the patient's perspective. International Journal of Obesity 24 (Suppl 3), S12-20.

The Pharmaceutical Services Negotiating Committee. 2004a: National Service Framework for Diabetes a guide for community pharmacists. UK: Ushers Print and Design.

The Pharmaceutical Services Negotiating Committee. 2004b: The new NHS Community Pharmacy Service - a summary of the structure. Retrieved 18 May 2006, from http://www.psnc. org.uk/uploaded_txt/Pharmacy_contract_summary_(Dec\% 2004).pdf.

The St Vincent Declaration. 1990: Diabetes Care and Research in Europe. The St Vincent Declaration. Diabetic Medicine 7,360.

Wermeille, J., Bennie, M., Brown, I. and McKnight, J. 2004: Pharmaceutical care model for patients with type 2 diabetes: integration of the community pharmacist into the diabetes team - a pilot study. Pharmacy World and Science 26, 18-25. 